\title{
Correlation between Inflammatory Biomarkers and Red Blood Cell Life Span in Chronic Hemodialysis Patients
}

\author{
Jie Ma ${ }^{a, b}$ Yanna Dou ${ }^{a, c}$ Hanjie Zhang ${ }^{a}$ Stephan Thijssen ${ }^{a}$ \\ Schantel Williams $^{\text {a }}$ Viktoriya Kuntsevich ${ }^{d}$ Georges Ouellet $^{\text {a, e }}$ \\ Michelle M.Y. Wong ${ }^{a, f}$ Vanja Persic ${ }^{a, g}$ Anja Kruse ${ }^{a, h} \quad$ Laura Rosales $^{a}$ \\ Yuedong Wangi Nathan W. Levin ${ }^{\mathrm{a}}$ Peter Kotanko ${ }^{\mathrm{a}, \mathrm{d}}$ \\ ${ }^{a}$ Renal Research Institute, New York, NY, USA; ${ }^{b}$ Department of Nephrology, Peking Union Medical College Hospital, \\ Chinese Academy of Medical Sciences and Peking Union Medical College, Beijing, and 'The First Affiliated Hospital \\ of Zhengzhou University, Henan, China; ${ }^{d}$ Icahn School of Medicine at Mount Sinai, New York, NY, USA; ${ }^{e}$ Hôpital \\ Maisonneuve-Rosemont, Montréal, QC, and f Department of Medicine, University of British Columbia, Vancouver,

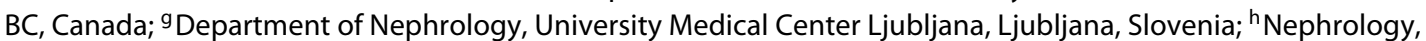 \\ Spitalzentrum Biel, Biel, Switzerland; 'Department of Statistics and Applied Probability, University of California, \\ Santa Barbara, CA, USA
}

\section{Key Words}

Red blood cell life span · Inflammation · Interleukins .

Uric acid · Hemodialysis · Anemia

\begin{abstract}
Background and Objectives: The pathogenesis of anemia in hemodialysis (HD) patients is dependent on multiple factors, with decreased red blood cell life span (RBCLS) being a significant contributor. Although the impact of reduced RBCLS on anemia is recognized, it is still a subject that is not well researched. The objective of this study was to investigate the relationship between RBCLS and inflammatory biomarkers in chronic HD patients. Design, Setting, Participants, and Measurements: RBCLS was calculated from alveolar carbon monoxide concentrations measured by gas chromatography. Interleukins (IL) IL-6, IL-18, IL-10, and high sensitivity $\mathrm{C}$-reactive protein were measured using bead-based multiplex assay. Measurements were carried out at baseline and
\end{abstract}

(c) 2017 S. Karger AG, Basel during follow-up. The associations between RBCLS and inflammatory biomarkers were evaluated using linear mixed effects models. Results: RBCLS measurements were available for $54 \mathrm{HD}$ patients. Their average age was $58.5 \pm 14.4$ years, $68.5 \%$ were males, $48.1 \%$ were diabetics, and the HD vintage was $51 \pm 48$ months. In 4 patients, RBCLS was measured once, while in 50 patients, up to 5 repeated RBCLS measurements were available. RBCLS was $73.2 \pm 17.8$ days (range 37.7-115.8 days). No association was found between RBCLS and any of the inflammatory biomarkers. Of note, RBCLS was positively correlated with levels of uric acid $(p=0.02)$ and blood urea nitrogen (BUN; $p=0.01$ ), respectively. Conclusion: Our study suggests that inflammation pathways reported by these biomarkers only have a limited role in causing premature RBC death. The positive correlation with uric acid and BUN warrants further studies.

(c) 2017 S. Karger AG, Basel

\section{KARGER}

E-Mail karger@karger.com

www.karger.com/bpu
Peter Kotanko, MD, FASN

Renal Research Institute

315 East 62nd Street, 4th Floor

New York, NY 10065 (USA)

E-Mail pkotanko@ rriny.com 


\section{Introduction}

Anemia is a typical complication of chronic kidney disease. The pathogenesis of anemia in hemodialysis (HD) patients is dependent on multiple factors, including insufficient production of endogenous erythropoietin (EPO), functionaliron deficiency, acute and chronicinflammation, hyperparathyroidism, aluminum toxicity, deficiency of nutrients such as folate and others, inhibition of erythropoiesis [1], increased apoptosis of erythroblasts [2], and shortened red blood cell life span (RBCLS). Previous research has indicated that RBCLS in chronic HD patients was reduced to approximately 60-90 days [3]. While exogenous erythropoiesis stimulating agents (ESA) are used successfully in the treatment of anemia in a majority of HD patients, some patients require very high ESA doses, a clinical phenotype called ESA resistance. While ESA resistance is frequently attributed to inflammation, functional iron deficiency and circulating EPO inhibitors, a shortened RBCLS has hardly been recognized as a contributing factor $[3,4]$. Inflammation and oxidative stress frequently co-exist in the maintenance HD patients [5] and their interconnection induces anemia complicated with EPO hyporesponsiveness [6]. HD patients present frequently with high levels of inflammatory markers such as high sensitivity C-reactive protein (hsCRP), interleukin (IL)-6, tumor necrosis factor- $\alpha$, neutrophil-to-lymphocyte ratio (NLR) [7], and lowlevels of serum albumin [8]. Increased levels of pro-inflammatory cytokines reduce the production of erythroid progenitor cells and this contributes to hyporesponsiveness to ESAs and poor treatment outcomes $[9,10]$. Elevated levels of inflammatory cytokines also contribute to functional iron deficiency. While the relationship between inflammation and renal anemia is well explored $[11,12]$, the impact of reduced RBCLS although recognized is not yet well researched.

The objective of this study was to further investigate the relationship between RBCLS and inflammatory biomarkers in chronic HD patients.

\section{Materials and Methods}

\section{Study Participants and Study Design}

We enrolled non-smoking HD patients from an urban HD center, and measured their RBCLS up to 5 times during a maximum follow-up of 18 months in a prospective observational study. ESA dosages were adjusted by the attending nephrologist to maintain hemoglobin (Hgb) levels within the range 10.0-11.9 g/dl. We excluded patients with hemorrhagic disorders, chronic lung disease, infection or cancer.

This study was conducted in accordance with the ethical principles set forth in the Helsinki Declaration, and approval was ob- tained from the New England institutional review board. Written informed consent was obtained from all individuals prior to their participation in this study.

\section{Data Collection}

Clinical data, including age, gender, HD vintage, comorbidities, vascular access type, dialysis adequacy $(\mathrm{Kt} / \mathrm{V})$, routine laboratory data, and weekly EPO dose were extracted from the study database.

\section{Measurements}

Whole blood samples were drawn before the first HD session of the week (Monday or Tuesday), centrifuged when appropriate and analyzed at Spectra Laboratories, Rockleigh NJ, to assess a complete blood count, hs-CRP, blood urea nitrogen (BUN), albumin, iron status (iron, ferritin, transferrin saturation), uric acid, carnitine, as well as total and direct bilirubin. In addition, EDTA blood samples were centrifuged immediately after collection, and plasma was aliquoted and frozen at $-70^{\circ} \mathrm{C}$ for further batch analysis of the pro-inflammatory cytokines IL- 6 and IL-18 and the antiinflammatory cytokine IL-10. Plasma levels of IL- 6 and IL-10 were measured in duplicates using the MILLIPLEX ${ }^{\circledR}$ MAP High Sensitivity Human Cytokine Panel kit (Millipore Corp., Billerica, MA, USA) with Luminex ${ }^{\circledR} 100^{\mathrm{TM}}$ System (Luminex Corporation, Austin, Tex., USA). Quantitative analysis of plasma IL-18 was made in duplicates by high-sensitivity ELISA (MLB, Nagoya, Japan) that uses 2 monoclonal antibodies against 2 different epitopes of human IL-18 according to the instructions in the supplier manual. Body weight was measured before and after each HD session. The doses of EPO (epoietin alpha) are expressed as IU/kg body weight per week. We used the EPO resistance index as the metric of EPO resistance, defined as the weekly weight-adjusted EPO dose (U/kg/week) divided by Hgb level (g/dl) $[13,14]$.

\section{Measurement of Alveolar Carbon Monoxide Concentrations and Calculation of RBCLS}

Breakdown of heme results in the production of carbon monoxide (CO), and the alveolar CO concentration can be used to estimate RBCLS [15]. Alveolar breath samples were obtained twice using the GaSampler ${ }^{\mathrm{TM}}$ device (Quintron Instruments Milwaukee, WI, USA) before the first blood sample was drawn. A sample of ambient air from the patient's home was obtained with an open plastic bottle that was kept overnight in the patient's home to allow for equilibration of gases. CO concentration was measured by gas chromatography (Model 910, Buck Scientific, East Norwalk, CT, USA). Patient alveolar air, ambient air from the patient's home, and standard gas (20 parts per million) used for calibration were measured and the area under the curves (AUC) representing the $\mathrm{CO}$ concentration were reported. All gas samples were measured in triplicates, and the respective average AUC of each air sample were used to calculate the $\mathrm{CO}$ concentrations. The endogenous $\mathrm{CO}$ is the difference between the average alveolar and home ambient air CO concentrations. The endogenous CO concentration and RBCLS were computed as previously reported [15-17].

\section{Statistical Analyses}

Continuous variables are presented as mean \pm SD if normally distributed and as median (25th, 75th percentile) otherwise. Categorical variables are presented as percentages of the respective group.

Linear mixed effects (LME) models were used to explore the associations between RBCLS and independent variables. We used 
Table 1. Baseline characteristics

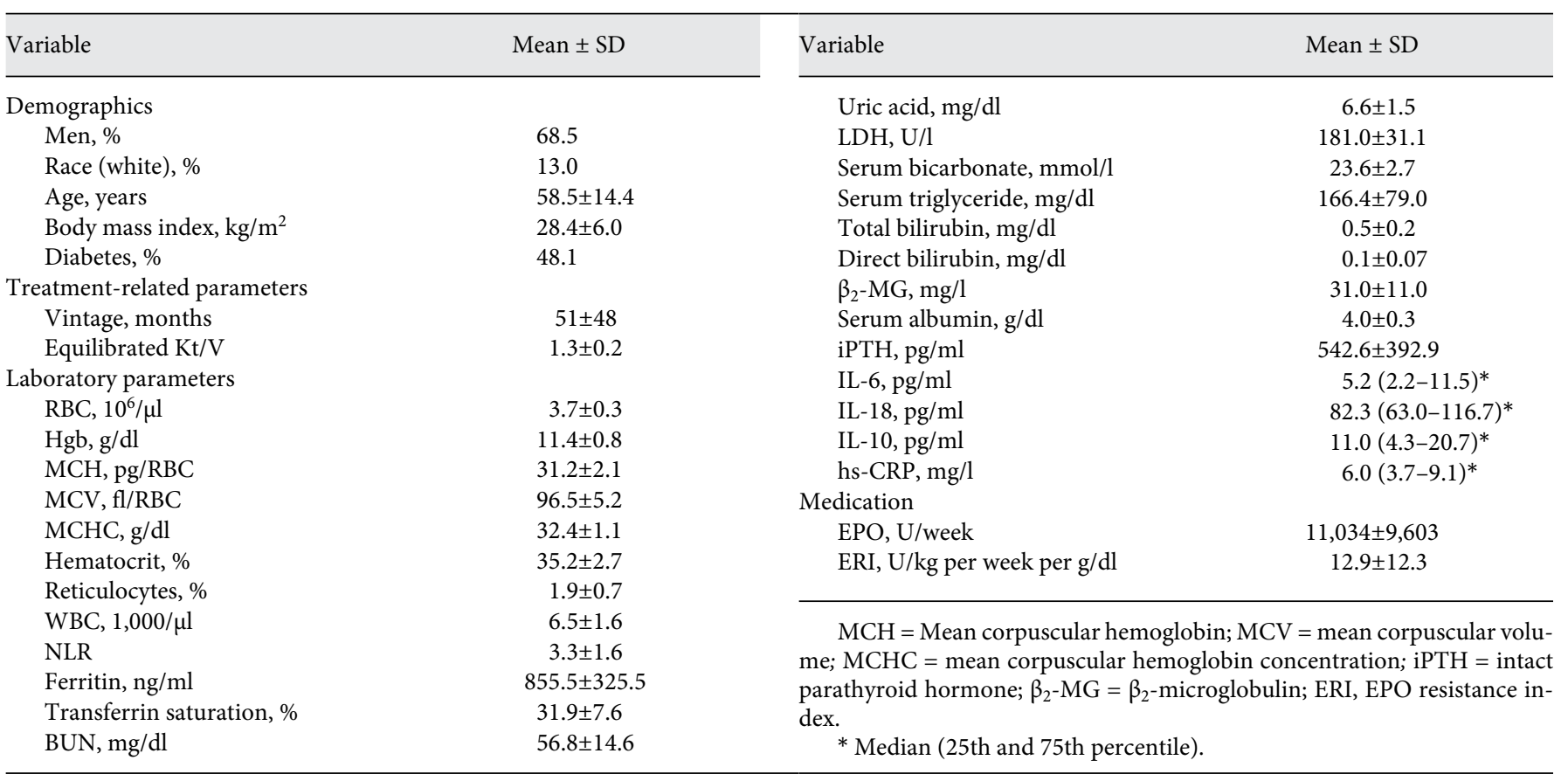

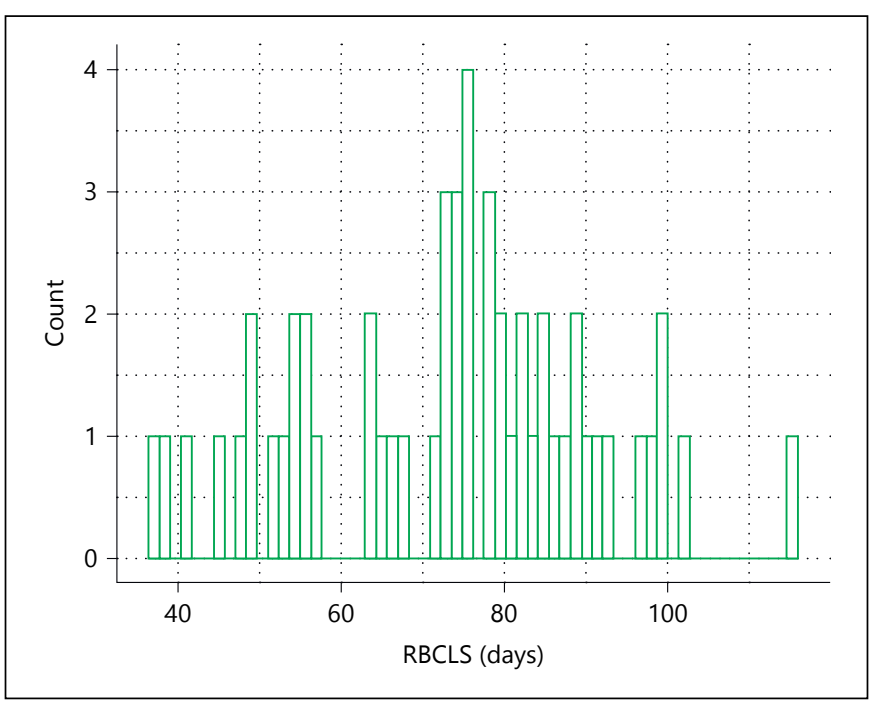

Fig. 1. Frequency distribution of RBCLS. In patients with multiple measurements, the measurements were averaged and these averages were included in the histogram. The mean RBCLS was $73.2 \pm$ 17.8 days (range 37.7-115.8 days).

simulations to estimate the power of our study with parameters set as the estimates from the real data.

Statistical analyses were performed using SAS version 9.3 (SAS Institute Inc., Cary, NC, USA) and R 3.0.2 (libraries ggplot2, nlme; R Foundation for Statistical Computing, Vienna, Austria).

\section{Results}

RBCLS measurements were available in $54 \mathrm{HD}$ patients. Their average age was $58.5 \pm 14.4$ years, $68.5 \%$ were males, $48.1 \%$ were diabetics, and the $\mathrm{HD}$ vintage was $51 \pm 48$ months (table 1). In 4 patients, RBCLS was measured once, while in 50 patients up to 5 repeated RBCLS measurements were available. Among these 50 patients, 7 patients had 2 measurements, 9 patients had 3 measurements, 12 patients had 4 measurements, and 22 had 5 measurements.

The mean RBCLS was $73.2 \pm 17.8$ days (range $37.7-$ 115.8 days; fig. 1). We did not observe any significant correlation between RBCLS and white blood cells (WBC), NLR, hs-CRP, IL-6, IL-18, and IL-10, respectively (fig. $2 \mathrm{a}-\mathrm{f}$ ).

Of note, RBCLS was positively correlated with uric acid $(p=0.02)$ and BUN $(p=0.01)$, respectively (fig. 3a, b).

\section{Discussion}

The key finding of this study is that there is lack of correlation between RBCLS and inflammatory indicators. To the best of our knowledge, this is the first study in chronic HD patients to test the hypothesis of a relationship between RBCLS and these inflammatory biomarkers. 


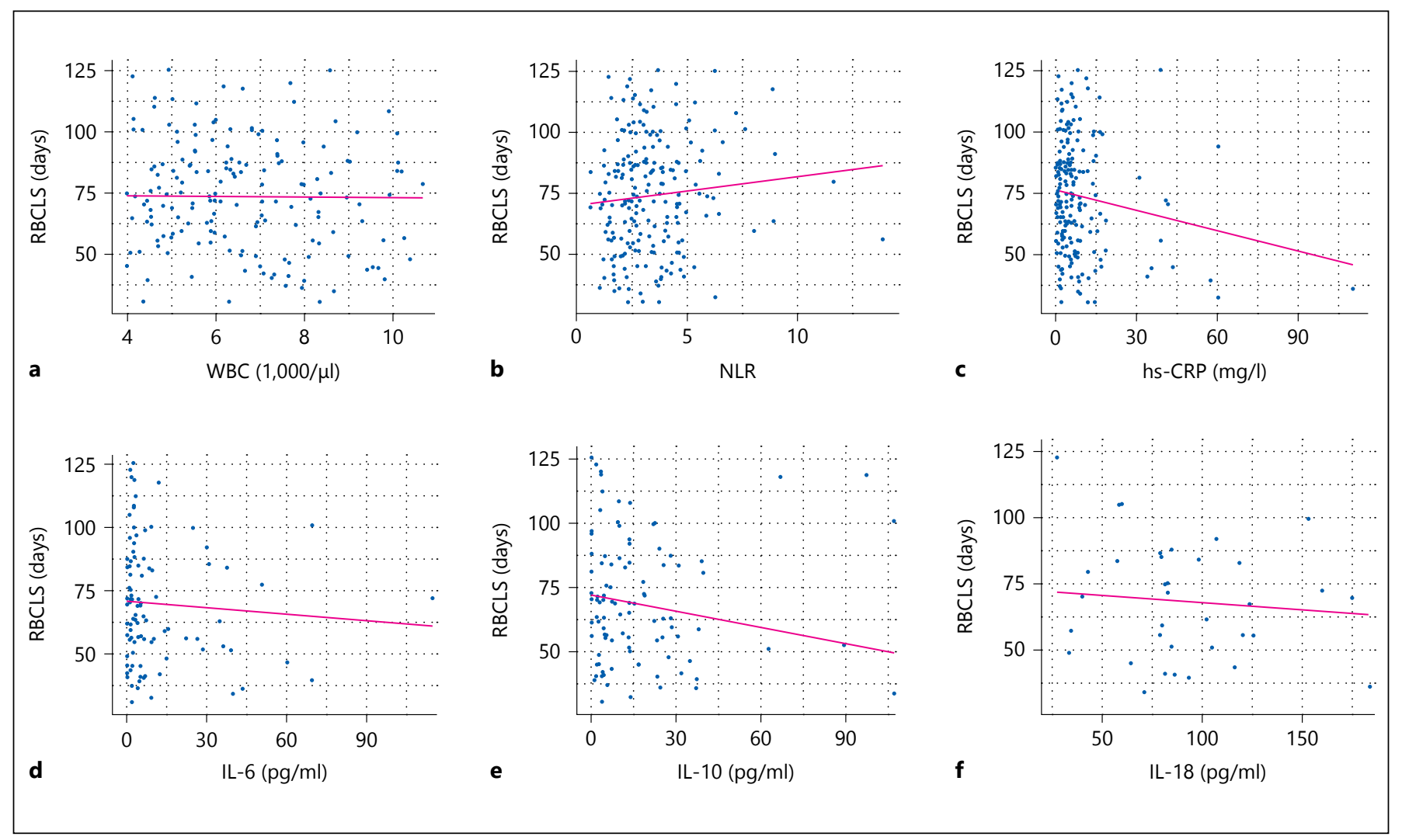

Fig. 2. Relationship between inflammatory biomarkers and RBC life span. Each data point is an individual measurement; there are multiple measurements per patient. The solid lines represent the fixed effect estimated by LME models. None of these associations was statistically significant. a WBC (beta $=-0.16, \mathrm{p}=0.89)$; $\mathbf{b}$ NLR (beta $=1.18, \mathrm{p}=0.35) ; \mathbf{c}$ hs-CRP (beta $=-0.27, \mathrm{p}=0.23) ; \mathbf{d}$ IL-6 $($ beta $=-0.09, \mathrm{p}=0.51) ; \mathbf{e}$ IL-10 (beta $=-0.21, \mathrm{p}=0.31) ; \mathbf{f}$ IL-18 (beta $=-0.05, \mathrm{p}=0.13$ ).

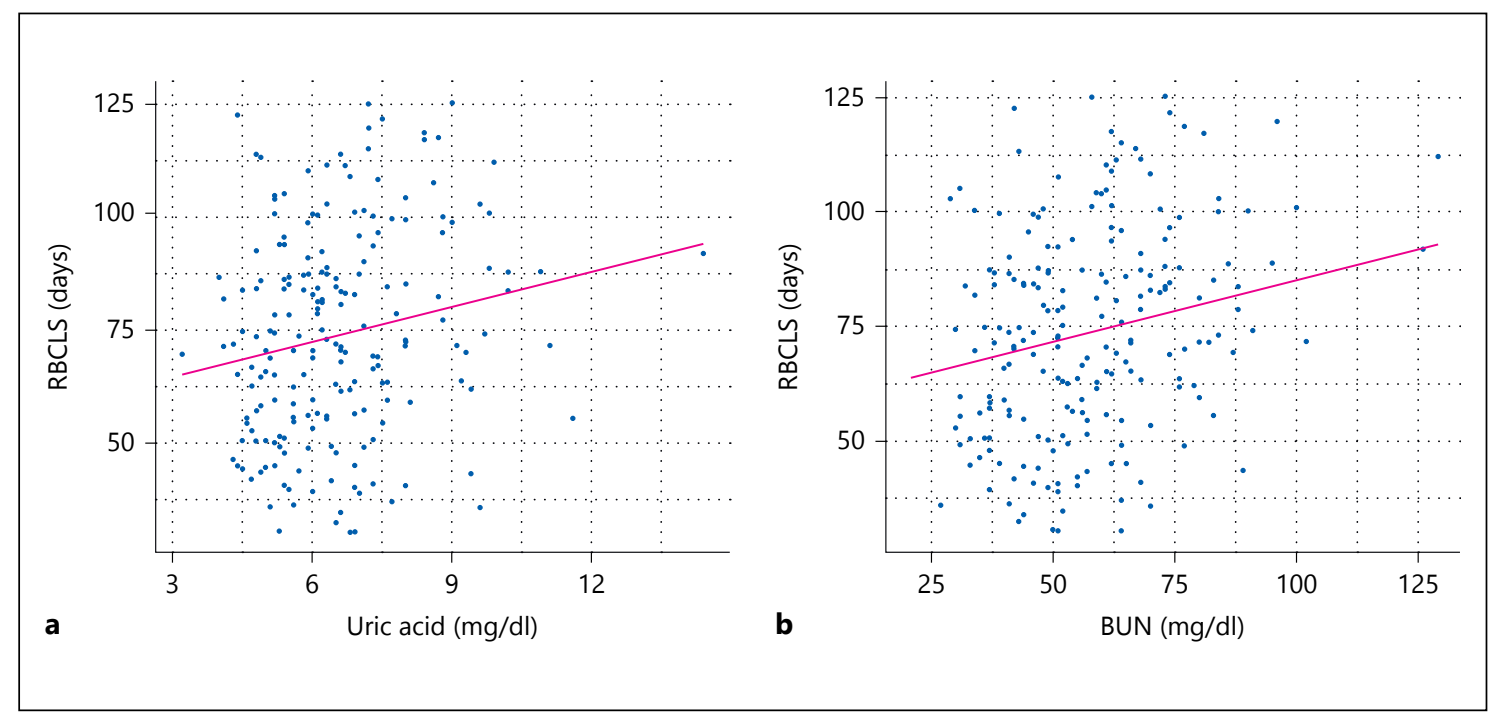

Fig. 3. Positive correlations between RBCLS and $(\mathbf{a})$ uric acid (beta $=2.60, \mathrm{p}=0.02)$ and $(\mathbf{b}) \mathrm{BUN}(\mathrm{beta}=0.27$, $\mathrm{p}=0.01$ ) levels. Each data point is an individual measurement there are multiple measurements per patient. The solid lines represent the fixed effect estimated by LME models. 
The relationship between inflammation and anemia in dialysis patients is well described and it has been shown that inflammation exerts both direct and indirect effects on erythropoiesis [18-20]. However, little is known about an association between inflammatory cytokines and RBCLS. In the present research, we studied about cytokines with known involvement in the genesis of anemia. IL-6 impairs late stages of erythroid development, after cells have been primed for Hgb synthesis [21]. In vitro data show a direct negative effect of IL-10 on erythroid progenitor cells proliferation and indicate that IL-10 could inhibit the growth of these cells [22]. A recent study shows that IL-10 induces the expression of heme oxygenase-1 [23], which may enhance heme degradation, resulting in an increase in the amount of iron within monocytes, and subsequent incorporation within the reticuloendothelial system and the development of anemia. IL-18, a pro-inflammatory cytokine with diverse pleiotropic effects, induces interferon gamma production by natural killer cells, T cells, and activated macrophages. IL-18 also regulates both T helper 1 (Th1) and Th2 responses, thus playing an important role in the inflammatory process [24].

While not being part of the primary data analysis, we found that uric acid and BUN were correlated with RBCLS. It is tempting to speculate that the positive correlation between uric acid levels and RBCLS may be related to its antioxidant actions. Our research also corroborates a recently reported positive relationship between BUN and RBCLS [3], and currently, the underlying biol- ogy of that finding remains elusive. The correlation of RBCLS with uric acid and BUN warrants further studies.

We used the CO method developed by Strocchi et al. [15] to measure RBCLS. The main advantages of this method are its noninvasive nature, the avoidance of radioactive label, and its practical simplicity. However, the underlying principle of that method deemed it necessary to exclude smokers from this research, and this certainly was a limitation of this study.

Whenever the results of a study comprise negative findings, considerations of statistical power and sample size are important. While being one of the largest studies with repeated measurements of RBCLS in chronic HD patients, its statistical power is still low. With estimated parameters as the true parameters, our study has only $20 \%$ power at the significance level of $5 \%$ for NLR as a marker. To have $80 \%$ of power, one would need a likely prohibitively large number of 500 patients. The nonsignificant associations may be a result of small sample size and large variation between patients.

In conclusion, our study did not find an association between RBCLS and inflammatory biomarkers in chronic HD patients. This suggests that inflammation only has a limited role in the genesis of premature $\mathrm{RBC}$ death.

\section{Disclosure Statement}

P.K. holds stock in Fresenius Medical Care. M.M.Y.W. is a research consultant with Arbor Research Collaborative for Health.

\section{References}

1 Weiss G, Goodnough LT: Anemia of chronic disease. N Engl J Med 2005;352:1011-1023.

2 Sitter T, Bergner A, Schiffl H: Dialysate related cytokine induction and response to recombinant human erythropoietin in haemodialysis patients. Nephrol Dial Transplant 2000;15:1207-1211.

3 Sato Y, et al: Shortened red blood cell lifespan is related to the dose of erythropoiesis-stimulating agents requirement in patients on hemodialysis. Ther Apher Dial 2012;16:522-528.

4 Dou Y, et al: Red blood cell life span and 'erythropoietin resistance'. Kidney Int 2012; 81:1275-1276; author reply 1276 .

5 Esgalhado M, et al: Effect of acute intradialytic strength physical exercise on oxidative stress and inflammatory responses in hemodialysis patients. Kidney Res Clin Pract 2015; 34:35-40.

6 Khalil SK, et al: Oxidative stress during erythropoietin hyporesponsiveness anemia at end stage renal disease: molecular and biochemical studies. J Adv Res 2016;7:348-358.
7 Malhotra R, et al: Relationship of neutrophilto-lymphocyte ratio and serum Albumin levels with C-reactive protein in hemodialysis patients: results from 2 International cohort studies. Nephron 2015;130:263-270.

8 Costa E, et al: Neutrophil activation and resistance to recombinant human erythropoietin therapy in hemodialysis patients. Am J Nephrol 2008;28:935-940.

9 de Francisco AL, Stenvinkel P, Vaulont S: Inflammation and its impact on anaemia in chronic kidney disease: from haemoglobin variability to hyporesponsiveness. NDT Plus 2009;2(suppl_1):i18-i26.

10 Won HS, et al: IL-6 is an independent risk factor for resistance to erythropoiesis-stimulating agents in hemodialysis patients without iron deficiency. Hemodial Int 2012;16: 31-37.

11 Pancha Mbouemboue O, et al: Frequency of specific cardiovascular disease risk factors among Cameroonian patients on dialysis: the cases of anaemia, inflammation, phosphate, and calcium. Cardiol Res Pract 2016;2016: 5031927.

12 de Oliveira Junior WV, et al: Inflammation and poor response to treatment with erythropoietin in chronic kidney disease. J Bras Nefrol 2015;37:255-263.

13 Tsai MT, Liu HC, Huang TP: The impact of malnutritional status on survival in elderly hemodialysis patients. J Chin Med Assoc 2016;79:309-313.

14 Harrison LE, et al: Endotoxaemia in haemodialysis: a novel factor in erythropoetin resistance? PLoS One 2012;7:e40209.

15 Strocchi A, et al: A simple carbon monoxide breath test to estimate erythrocyte turnover. J Lab Clin Med 1992;120:392-399.

16 Mitlyng BL, et al: Use of breath carbon monoxide measurements to assess erythrocyte survival in subjects with chronic diseases. Am J Hematol 2006;81:432-8.

17 Furne JK, et al: Simplification of the end-alveolar carbon monoxide technique to assess erythrocyte survival. J Lab Clin Med 2003;142:52-57. 
18 Barany P, Divino Filho JC, Bergstrom J: High C-reactive protein is a strong predictor of resistance to erythropoietin in hemodialysis patients. Am J Kidney Dis 1997;29: 565-568.

19 Locatelli F, et al: Nutritional-inflammation status and resistance to erythropoietin therapy in haemodialysis patients. Nephrol Dial Transplant 2006;21:991-998.
20 Gunnell J, et al: Acute-phase response predicts erythropoietin resistance in hemodialysis and peritoneal dialysis patients. Am J Kidney Dis 1999;33:63-72.

21 Wu F, Satchwell TJ, Toye AM: Anion exchanger 1 in red blood cells and kidney: Band 3's in a pod. Biochem Cell Biol 2011;89:106-114.

22 Geissler K, et al: Interleukin-10 inhibits erythropoietin-independent growth of erythroid bursts in patients with polycythemia vera. Blood 1998;92:1967-1972.
23 Lee TS, Chau LY: Heme oxygenase-1 mediates the anti-inflammatory effect of interleukin-10 in mice. Nat Med 2002;8:240246.

24 Anyona SB, et al: Functional promoter haplotypes of interleukin-18 condition susceptibility to severe malarial anemia and childhood mortality. Infect Immun 2011;79:49234932 . 